\title{
Omission training and positive conditioned suppression in the rat
}

\author{
C. M. LOCURTO, JOHN TIERNEY, and STEPHEN FITZGERALD \\ College of the Holy Cross, Worcester, Massachusetts 01610
}

\begin{abstract}
Suppression of operant responding during a conditioned stimulus (CS) was studied in two procedures. In both procedures, operant leverpressing was maintained by a variable-interval 1-min food-delivery schedule, and insertion of a second lever served as the CS. In the first procedure, autoshaping, food followed each CS presentation irrespective of a subject's behavior during the CS. In the second procedure, omission training, contact with the CS canceled the delivery of food scheduled for the end of that CS. In the first experiment, subjects were exposed to omission training followed by autoshaping; these procedures were reversed in the second experiment. In each experiment, the omission contingency resulted in fewer CS contacts and less suppression of operant responding during the CS than did autoshaping. These differences were more notable in subjects receiving the sequence autoshaping $\rightarrow$ omission training (Experiment 2). Direct observations in Experiment 2 revealed that, for subjects that were contacting the CS frequently when the omission contingency was introduced, reductions in signal contacts were accompanied by redistributions of behavior. The form of these redistributions depended upon behavior allocation at the time the omission contingency was imposed.
\end{abstract}

Few phenomena have proven so intriguing in recent years as the demonstration that hungry pigeons will continue to peck a visual conditioned stimulus (CS) when the sole consequence is to cancel food delivery. As first demonstrated by Williams and Williams (1969), this omission training procedure arranged for food to follow the offset of every CS presentation as long as the CS was not pecked. The persistence of CS-pecking was remarkable in that nearly half of the scheduled unconditioned stimuli (US) were lost during extended training. This finding hampers an interpretation of autoshaping that claims that CSdirected responding is maintained by the adventitious relation between responding and the US (cf. Brown \& Jenkins, 1968, p. 7). Moreover, it has served as the foundation for theoretical interpretations of conditioned behavior that emphasize Pavlovian contingencies (e.g., Hearst \& Jenkins, 1974; Lajoie \& Bindra, 1976; Peden, Browne, \& Hearst, 1977).

Despite the impact of this demonstration, several problems suggest that the use of the omission procedure as an analytic tool is not without limitations. Some of these problems are logical, restricting the conclusions that may be drawn from this procedure, and have been discussed elsewhere (e.g., Boakes, 1977; Jenkins, 1977; Locurto, 1980; Locurto, Duncan,

This work was supported in part by Grant R03MH 30419 from the National Institute of Mental Health and by funds granted by the Committee on Research and Publication, College of the Holy Cross. Requests for reprints should be addressed to the first author, Department of Psychology, College of the Holy Cross, Worcester, Massachusetts 01610.
Terrace, \& Gibbon, 1980). Other problems concern the generality of the omission phenomenon. For example, when water is used instead of food as the US, pigeons' autoshaped keypecking rapidly falls to low levels under an omission contingency (Woodruff \& Williams, 1976). Similarly, in species other than the pigeon, the omission procedure supports levels of responding no greater than those observed during extinction or a random-control procedure (rat-Locurto, Terrace, \& Gibbon, 1976, 1978; crow-Powell, Kelly, \& Santisteban, 1975; monkey-Gamzu \& Schwam, 1974; guinea pig-Poling \& Poling, 1978; see Locurto, 1980 , for a review).

Substantial variability among species poses the question of how to interpret these different levels of omission responding. In the case of the persistence of the pigeon's keypeck under an omission procedure using a food US, it is reasonable to assume that the CS retains excitatory strength despite the negative response-US contingency. Yet, how is one to evaluate low levels of responding under an omission procedure? At least two interpretations are plausible. Low response levels may indicate that the CS no longer controls conditioned behavior (i.e., it has become irrelevant) or that conditioned behavior has been redirected (i.e., the CS retains control over behavior that has been displaced from the CS).

Several studies have provided evidence pertinent to this issue. Barrera (1974) noted that although pigeons' signal-directed responding was substantially reduced during omission training, pecking per se continued at high levels but was redirected away from the response key (see, also, Lucas, 1975). Similarly, Boakes 
(1977, 1979) noted that, during autoshaping, some rats directed their behavior primarily at an illuminated lever that served as the CS (sign-tracking), while other rats approached the site of food delivery (goal-tracking). Rats that predominantly sign-tracked were then placed on an omission procedure in which leverpresses canceled food, while rats in which goaltracking predominated experienced an omission procedure wherein contact with the food-delivery site canceled food. Both omission contingencies substantially reduced the target response. In some, but not all, cases, suppression of the target response resulted in an increase in the second response (e.g., if goaltracking defined the omission contingency, signtracking increased).

This work has been extended by Holland (1979) in a study of the susceptibility of different responses in the rat (viz., rearing, startle responses, head-jerks, goal-tracking) to an omission procedure using visual or auditory CSs. These responses varied in their sensitivity to the omission contingency. In some, but not all, instances, reductions in the target behavior led to increases in other responses. Similar results have been noted in the pigeon (Morrison, 1974, cited in Schwartz \& Gamzu, 1977; Woodruff \& Williams, 1976).

These data indicate that the imposition of an omission contingency for one response may increase the frequency of other responses (see Dunham, 1971, for similar conclusions regarding punishment). Yet, these data do not necessarily reveal that the CS retains the same strength to control behavior following substantial reductions in the target response. It is likely, as Boakes (1977) noted, that some behavior will take the place of a frequently occurring response that has been suppressed. Whether these compensatory responses reflect equivalent CS control cannot be determined from observations of this type. The mere presence of other behavior following reductions in the target response gives an incomplete picture of the current strength of the CS.

To explore this issue further, the strategy of the present work was to observe the course of omission training in the rat in the context of a positive conditioned suppression procedure. In this procedure, CS-US pairings are superimposed, independently of a subject's behavior, upon a baseline of appetitive operant responding. Under appropriate temporal parameters, operant responding is reliably reduced during Pavlovian trials (e.g., Azrin \& Hake, 1969), raising the possibility that the suppression of operant responding during these trials may be accounted for by the occurrence of autoshaped behavior directed at the CS and/or site of food delivery (see Karpicke, 1978; Karpicke, Christoph, Peterson, \& Hearst, 1977; Schwartz, 1976). In this study, the suppression procedure served a different purpose: The magnitude of suppression of operant responding was taken as an index of the current strength of the CS during both standard Pavlovian trials (i.e., autoshaping, US delivered at the end of each CS irrespective of responding) and omission training (i.e., US followed CS only when contact with the CS did not occur).

In Experiment 1, rats were exposed to an omission procedure followed by autoshaping. In Experiment 2, this sequence was reversed and detailed observations were made of subjects' behavior during autoshaping followed by omission training.

\section{EXPERIMENT 1}

\section{Method}

Subjects. Four experimentally naive male hooded rats, approximately 130 days old at the start of training, were maintained at $80 \%$ of their free-feeding weights throughout the experiment.

Apparatus. Each experiment was conducted in a standard test chamber (Lehigh Valley, Inc.). The subject's compartment measured $31 \mathrm{~cm}$ wide $\times 27 \mathrm{~cm}$ high $\times 26 \mathrm{~cm}$ deep. The test panel, painted flat black, contained a food magazine $1 \mathrm{~cm}$ above the floor and two retractable levers (BRS/LVE RR 1-005) located $7.5 \mathrm{~cm}$ to either side of the magazine. A houselight that operated continuously throughout each session was $21 \mathrm{~cm}$ above the magazine. A speaker, mounted behind the test panel on the wall of the sound-attenuating shell, delivered white noise continuously. Each lever, when inserted, protruded $2.5 \mathrm{~cm}$ into the chamber. The lever to the left of the magazine served as the operandum and remained in the chamber throughout all sessions. The lever to the right of the magazine required $2.5 \mathrm{sec}$ to be inserted and was flush with the test panel when not inserted. Insertion of this lever served as the $\mathrm{CS}$, and contacts with this lever were recorded via a drinkometer circuit (Lehigh Valley Electronics). Forty-five-milligram food pellets (P. J. Noyes, Formula A) served as both the operant reinforcer and US. Electromechanical equipment in an adjoining room programmed all procedures.

Procedures. Subjects were trained to press the left lever by the method of successive approximations. The schedule of reinforcement associated with that lever was lengthened gradually over a 7-day period to a variable-interval 1-min schedule (VI 1-min). Intervals were determined by the Fleshler-Hoffman (1962) formula. Subjects received 15 additional days of training at this value. Then baseline sessions were conducted by inserting the right lever for trials of $8 \mathrm{sec}$ according to a variable-time 1 -min schedule (VT 1 -min). Reinforcers were available for responses on the left lever during baseline trials, but no USs followed retraction of the right lever. Baseline sessions continued until two criteria were met: The proportion of right-lever insertions with at least one levercontact was no greater than .20 , and suppression ratio approached .50 for each subject. Suppression ratio was the ratio of left leverpresses during the $C S(B)$ to total left leverpresses during the $C S$ and during an 8-sec period immediately preceding trials [A;thus, $B /(A+B)]$. Ratios of approximately .50 indicate little change in leverpressing during the CS. Lower ratios indicate suppression.

When subjects had met both criteria, omission training was initiated. During this training, which continued for 40 sessions, each presentation of the right lever was followed by a food pellet unless the lever was contacted. The first lever-contact canceled the food pellet scheduled for the end of that trial but did not affect trial duration. Following the last omission training session, 25 sessions of autoshaping were conducted. During autoshaping, a US followed the end of each lever insertion.

Sessions consisted of 50 total food pellets, a criterion that produced a varying number of trials per session during omission train- 
ing, with a range of $15-30$ trials across subjects. Sessions were conducted 5 to 6 days each week.

\section{Results}

Figure 1 shows the suppression ratio for each subject during the 40 sessions of omission training and the subsequent 25 autoshaping sessions. There was a gradual decrease in the suppression ratio of each subject with extended omission training. Ratios averaged .46 during the first three sessions and .28 over the final three sessions $[\mathrm{t}(3)=17.90, \mathrm{p}<.01]$. For three subjects $(9,10,11)$, suppression was relatively constant over the final 20 sessions of omission training. During autoshaping, suppression ratios decreased to an average of .18 over the final three sessions. The suppression ratio of each subject was somewhat lower during the last three autoshaping sessions than during the final three omission training sessions, although this difference failed to achieve statistical significance $[\mathrm{t}(3)=2.31, .05<\mathrm{p}<.10]$.

Figure 2 shows the probability of contact with the right lever, indexed as the proportion of trials with at least one contact. The response probability of each subject increased early in omission training. Three of the four subjects reached their highest probability, which exceeded .50 for each subject, during the first 12 sessions. For a fourth subject (12), response probability generally remained at levels exceeding .70 dur- ing the first 25 sessions. With extended training, contact probability decreased substantially for each subject. Over the final three omission training sessions, response probability averaged .13. During subsequent autoshaping, the response probability of each subject increased to an average of .38 over the final three autoshaping sessions, a value reliably higher than that recorded over the last three sessions of the omission procedure $[\mathrm{t}(3)=3.08, \mathrm{p}<.05]$.

\section{Discussion}

The trend in CS contacts during omission training, an initial rise in response probability followed by decreases, parallels the results of previous autoshaping procedures using rats (e.g., Locurto et al., 1976, Experiment 1 ). The finding that operant responding remained moderately suppressed during the $\mathrm{CS}$ although CS contacts fell to low levels suggests that the rat's behavior, while redirected away from the CS, remained at least partially under CS control. To study this possibility further, detailed observations were made of subjects' behavior during autoshaping and omission training in the next experiment.

Experiment 2 also served to evaluate the effect of the sequence of treatments used in this study. Several studies have noted that prior exposure to omission training retarded subsequent performance under an autoshaping procedure (in pigeons: Barrera, 1974; in

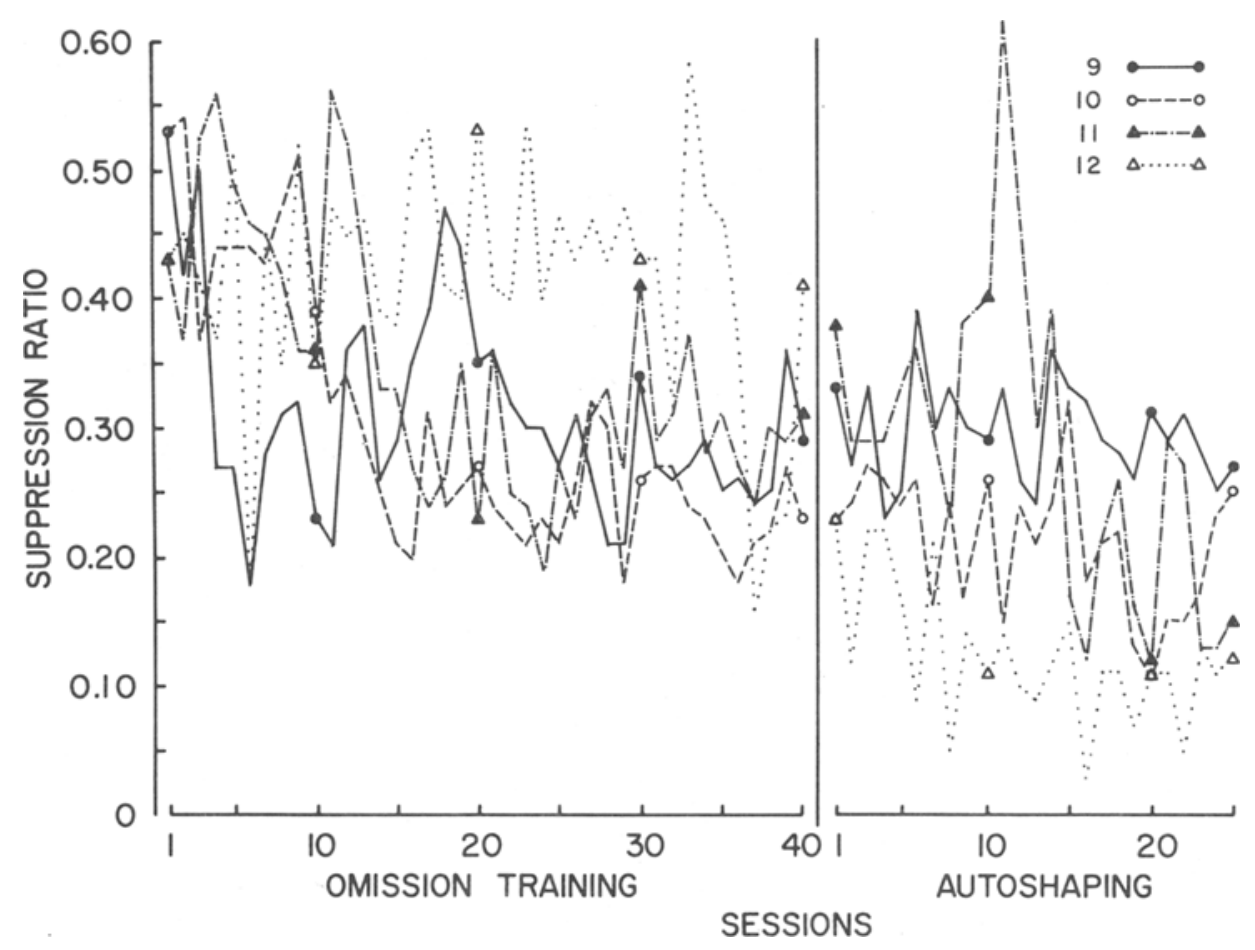

Figure 1. Suppression ratio for each subject in Experiment 1 during the 40 sessions of omission training and the 25 autoshaping sessions. 


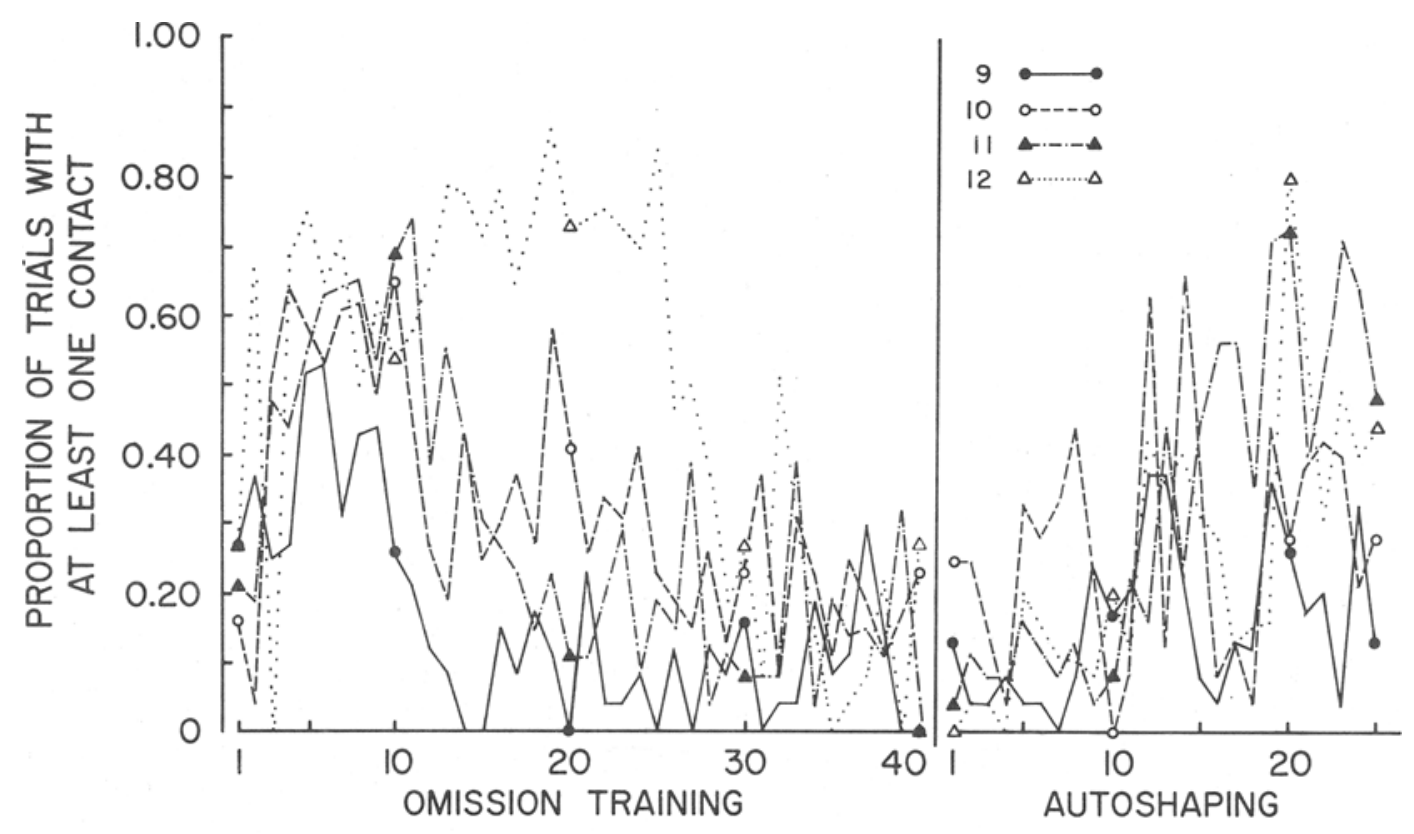

SESSIONS

\begin{abstract}
Figure 2. Probability of a contact with the CS for each subject, expressed as the proportion of trials with at least one contact, during the 40 sessions of omission training and the 25 autoshaping sessions of Experiment 1.
\end{abstract}

rats: Locurto et al., 1976, Experiment 2). In this study, CS contacts during autoshaping increased to moderate levels, while operant responding during CS presentations was not significantly altered. Although there is no previous evidence regarding the effects of these procedures on conditioned suppression, in studies wherein autoshaping constituted the only treatment, CS contact probabilities typically approached $\mathbf{1 . 0}$ (e.g., Locurto et al., 1976). In the second experiment, subjects received 15 autoshaping sessions prior to omission training.

\section{EXPERIMENT 2}

\section{Method}

Subjects. Four experimentally naive male hooded rats, approximately 120 days old at the start of training, were maintained as in Experiment 1.

Apparatus. The same apparatus was used as in Experiment 1.

Procedure. Leverpressing was shaped and was followed by training as in Experiment 1. Following the last baseline session, subjects received 15 autoshaping sessions followed by 40 sessions of omission training. Videotapes were made during the last autoshaping session and again during the 15 th omission training session. Tapes were scored by two observers to determine the proportion of total CS time that each subject spent in front of the CS lever, the food magazine, the VI lever, or in any other part of the chamber. Observers depressed one of four keys while viewing the videotapes. Key depressions accumulated a counter in fifths of a second. A rat was judged to be in front of a specified part of the test panel (e.g., food magazine) only if it faced toward the panel. A subject was judged to be in front of another part of the panel when it crossed an imaginary midline separating two portions.

\section{Results}

Figure 3 shows the suppression ratios for each subject during the last five autoshaping sessions and during each omission training session. By the end of autoshaping, the operant responding of three subjects was suppressed (suppression ratio below .10), while for a fourth subject (16), responding was only moderately suppressed. During omission training, suppression ratios of three rats increased. The increase was appreciable during the first $\mathbf{3 0}$ sessions for two subjects $(13,15)$ but was thereafter followed by decrements. For a third subject (14), the increase was less dramatic. Subject 16's suppression ratio was unchanged during autoshaping and the first 30 sessions of omission training. Extended omission training attenuated these between-subjects differences. As a group, the increase in suppression ratio from the last three autoshaping sessions (mean $=.12$ ) to the last three omission training sessions (mean $=.32$ ) was reliable $[\mathrm{t}(3)=3.95, \mathrm{p}<.05]$.

Figure 4 shows the probability of CS contact during autoshaping and omission training. For three subjects, response probability averaged greater than .70 during autoshaping but fell to approximately .10 during extended omission training. Subject 16 responded at low levels during both autoshaping and omission training. There was little change in any subject's CS-contact probability over the final 25 omission training sessions. The reduction in CS-contact probability from the last three autoshaping sessions to the last three sessions of omission training was significant $[\mathrm{t}(3)=12.21, \mathrm{p}<.01]$. 


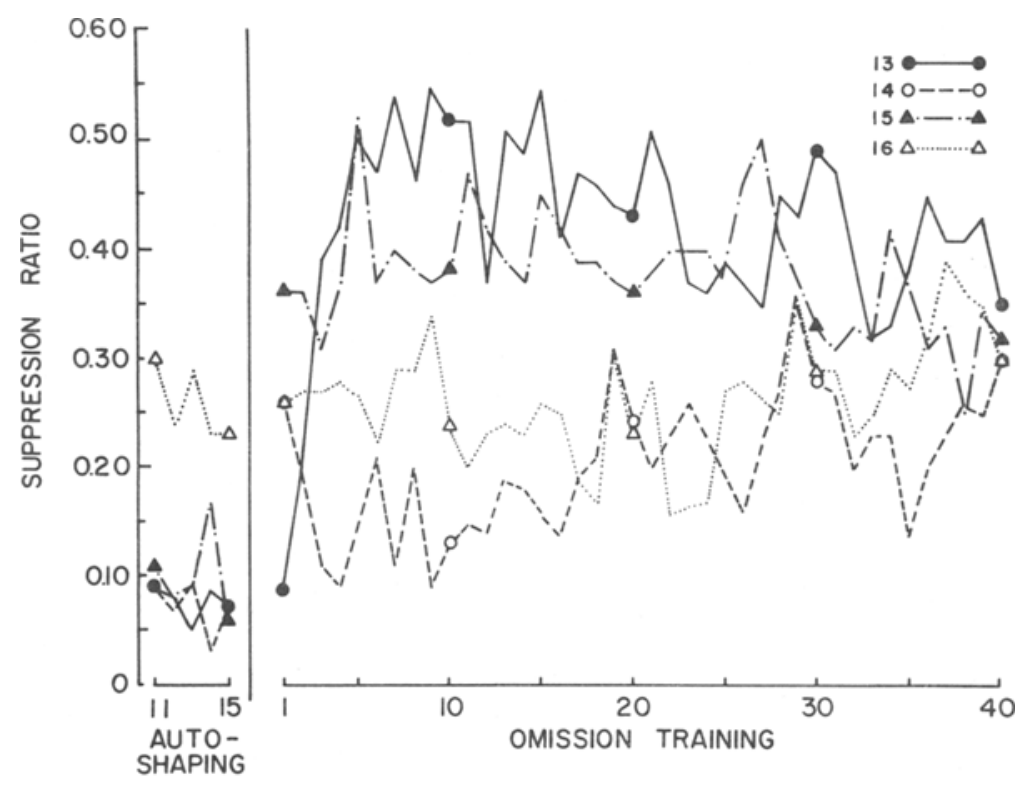

SESSIONS

Figure 3. Suppression ratio for each subject in Experiment 2 during the last five autoshaping sessions and the 40 sessions of omission training.
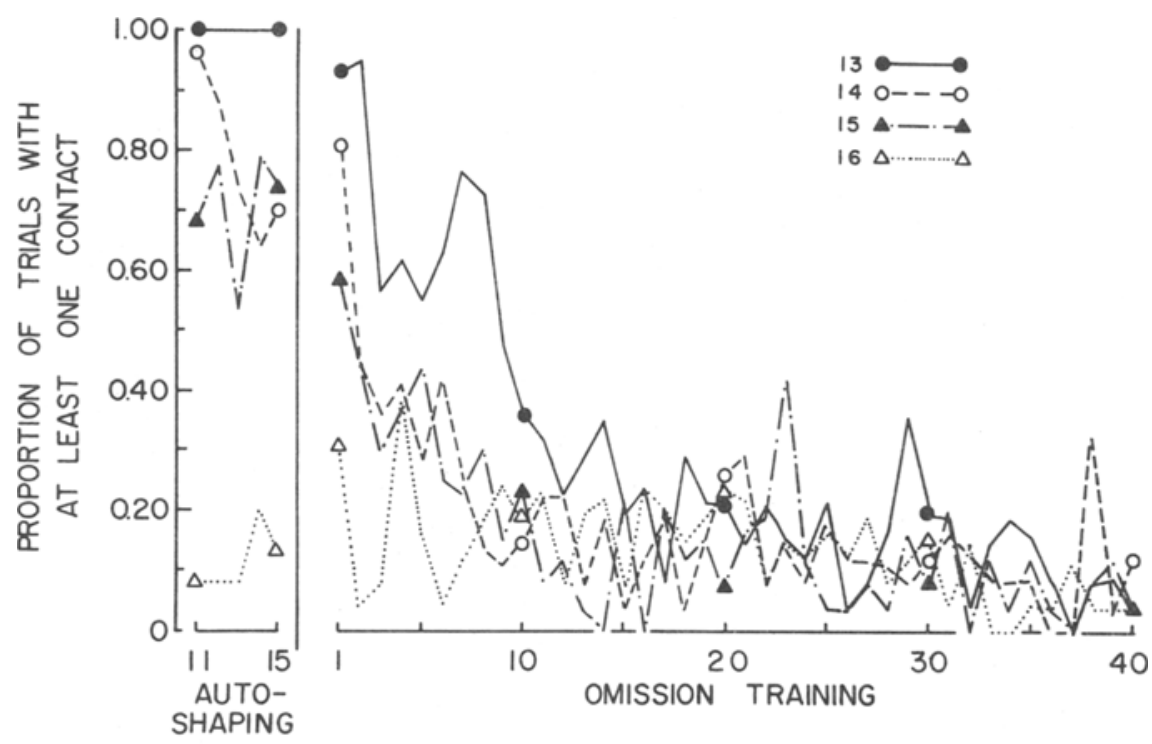

SESSIONS

Figure 4. Probability of a contact with the CS for each subject in Experiment 2 during the last five autoshaping sessions and the subsequent $\mathbf{4 0}$ sessions of omission training.

Comparison of Figures 3 and 4 reveals that, as in Experiment 1, suppression ratios during omission training were not well predicted by the probability of a CS contact. By Omission Session 15, the probability of a CS contact was low in all subjects (averaging only .17). The operant responding of two subjects remained moderately suppressed, whereas two other subjects $(13,15)$ evidenced little suppression.
Moreover, the reduction in differences between subjects with extended omission training was not accompanied by changes in CS-contact probability.

Observational data indicated that these individual differences were related to the distribution of a subject's behavior during trials. Figure 5 shows the proportion of trial time allocated to the VI lever, the food magazine, the CS lever, or to other areas of the 


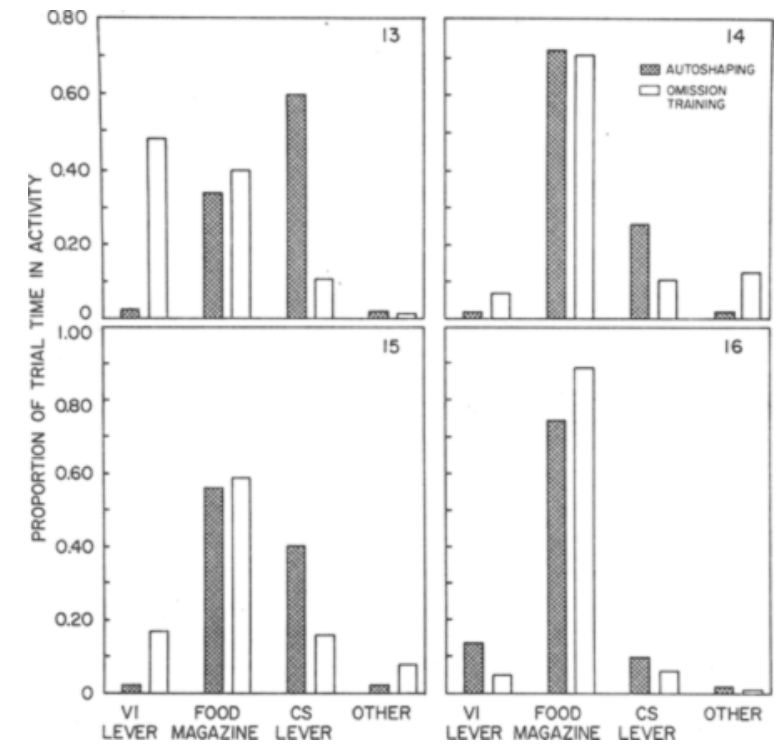

Figure 5. The proportion of time spent by each subject, during CS presentations, engaged in activities in various portions of the test chamber. Videotape recordings were made during the last autoshaping session and the 15th session of omission training in Experiment 2 and were scored by two trained observers.

test chamber during the 15 th autoshaping and omission training sessions. Hereafter, following Boakes (1977, 1979), time allocated to the food magazine and CS lever will be termed "goal-tracking" and "sign-tracking," respectively. This descriptive system captured over $90 \%$ of each subject's behavior during both autoshaping and omission training, since relatively little time was spent in any other portion of the chamber. Moreover, in three of four subjects (14, 15 , 16), goal-tracking predominated during both training procedures, even though, for two of these subjects $(14,15)$, the probability of at least one CS contact in a trial exceeded .70 during autoshaping (see Figure 4).

The behavior distributions of these subjects sort generally into three categories, which may be ranked according to the comparative strengths of sign- and goal-tracking. At one extreme, Subjects 13 and 15 evidenced the highest levels of sign-tracking among the four subjects during autoshaping. Not surprisingly, during subsequent omission training, the behavior distribution of these subjects showed the largest change, with sharp reductions in sign-tracking accompanied by increases in VI-lever time. Recall that the operant responding of these subjects was suppressed during autoshaping. Under an omission contingency, no suppression was evident for these subjects during the first 30 sessions, a pattern that reflects their redistribution of behavior. At the other extreme, Subject 16 allocated the lowest proportion of time to sign-tracking during autoshaping and was little affected by the imposition of an omission contingency. It is evident that this subject's moderate suppression during autoshaping and omission training was the outcome of persistent goal-tracking. Between these extremes was the behavior distribution of Subject 14, which included both high levels of goal-tracking and moderate levels of sign-tracking during autoshaping. Although the reduction in CS contacts for this subject was similar to that of Subjects 13 and 15 , the redistribution of behavior was less dramatic.

\section{GENERAL DISCUSSION}

These findings may be summarized as follows: (1) After extended omission training, CS contacts were reduced to low levels, while operant responding continued to be moderately suppressed during the CS. Thus, the CS may continue to control behavior incompatible with an ongoing operant following reductions in the target response. (2) A subject's initial adjustment to the omission contingency depended upon the distribution of behavior at the time the contingency was introduced.

These data, as well as the results of related experiments (e.g., Boakes, 1977, 1979; Holland, 1979) indicate that, to understand omission training, it is of fundamental importance to know the range of responses engendered by a particular CS-US combination and the factors that govern behavior redistribution during this procedure. Comparison of Experiments 1 and 2 suggests that one such factor may be prior experience. For experienced subjects, if signtracking were strong, a pronounced redirection of behavior followed the initiation of omission training (e.g., Subjects 13 and 15, Experiment 2). However, if alternative signal-controlled behavior was of sufficient strength, as was goal-tracking for Subject 14, a relatively less pronounced redistribution occurred, and the CS maintained behavior incompatible with the ongoing operant. For naive subjects, the omission contingency was not adequately sampled until relatively strong sign-tracking developed. Thus, signtracking must occur at moderate levels before the omission contingency decreases it and other behavior emerges under CS control. The pattern of signal-contact probability and conditioned suppression for subjects in Experiment 1 is consonant with this interpretation.

It must be added that extended exposure to the omission contingency produced comparable levels of suppression and CS-contact probabilities for subjects in both Experiments 1 and 2. Extended omission training also reduced the differences between subjects noted during the early portions of Experiment 2 . The attenuation of these differences suggests that, regardless of prior experience, an equilibrium condi- 
tion was reached eventually under these experimental parameters wherein behavior that substituted for the target response, such as goal-tracking, competed with the ongoing operant to yield moderate suppression.

Response substitution would also seem to be a function of the Pavlovian response system studied, that is, the CS-response-US combination employed. Holland (1979) noted that, in some instances, such as when an omission contingency was applied to goaltracking or rearing in the rat with a visual CS and a food US, reductions in the target response led to increases in other behavior. However, with an auditory $\mathrm{CS}$, the frequencies of goal-tracking and a head-jerk response were reduced during omission training, but no other behavior was observed to increase in frequency. To the extent that the emergence of other behavior reveals continued CS control, perhaps not all reductions in the target response in omission training should be interpreted in the same manner; some may indicate redirected behavior under CS control, and others may indicate reduced CS control. If so, to reveal the properties of the CS, it may be necessary to employ experimental strategies other than simply observing the strength of the target response. For example, in addition to the conditioned suppression procedure, one might ask whether the CS in omission training supports second-order conditioning or whether it can participate in compound conditioning to produce blocking (cf. Holland, 1977).

These results converge with related work concerning the utility of a behavioral competition framework in understanding omission training (Boakes, 1977, 1979; Holland, 1979) and the positive conditioned suppression procedure (e.g., Karpicke, 1978; Staddon, $1977 \mathrm{a}, 1977 \mathrm{~b}$ ). At present, we have relatively little information on this issue. There are cases in which behavioral competition appears to be inadequate (e.g., Dickinson \& Dearing, 1979; Gutman \& Maier, 1978), and others in which improper inferences may be drawn without an understanding of the kinds of directed behavior induced by experimental arrangements (Karpicke, 1978). At the very least, these factors constrain the generalizations that may be drawn, without reference to behavioral competition, concerning the outcome of pitting Pavlovian against operant relations.

It should also be recognized that these data do not permit one to decide whether the behavioral changes induced during omission training are the outcome of Pavlovian or operant processes. There are two findings to be explained: the reductions in the target response and the persistence of moderate levels of suppression. Either Pavlovian or operant mechanisms might account for these results. In Pavlovian terms, these changes are the result of stimulus differentiation (Dickinson \& Mackintosh, 1978): As responding is reduced to the CS, due to the fact that the CS is not followed by the US, other stimuli in the chamber more highly predictive of food-viz., the food magazine and VI lever-induce directed (e.g., autoshaped) behavior. Alternatively, behavior redistribution may be characterized in operant terms, as the result of the reinforcement of behavior (viz., goal-tracking and VI leverpressing) other than signal contacts. Stated another way, these behavioral adjustments may be considered either as a case of the emergence of other CSs or as the CS acquiring the function of an operant discriminative stimulus, shifting behavior away from the CS to avoid the loss of the US (i.e., "Do anything but contact the CS to be reinforced").

There is one caveat to this interpretation: Omission training not only introduces a negative response contingency, but also yields variations in the probability of the US at trial termination. However, it is doubtful that the present results can be ascribed to variations in US probability. In Experiment 2, for example, CS-contacts were at low levels by the 15 th session of omission training, so that food followed the CS on approximately $85 \%$ of trials. It is unlikely that the small difference in US probability between autoshaping and this point in omission training ( $100 \%$ vs. $85 \%$ ) accounted for the suppression ratio differences observed in Subjects 13 and 15 between these two procedures.

Previous research indicates that US probability per se does not govern the changes in behavior distribution noted here. Boakes, using a yoked design, observed that, while an omission contingency affected the behavior distribution of master subjects, there was little change in the responding of yoked subjects (see, also, Locurto et al., 1980; Locurto et al., 1978). Holland (1979) reported similar data from a yoked design. Yoked subjects did not display behavior redistribution under conditions that produced an increase in other behavior for omission subjects, viz., using either magazine-directed or rearing behavior as the target response in conjunction with a visual CS (see Gibbon, Farrell, Locurto, Duncan, $\&$ Terrace, 1980, for a parametric evaluation of probability effects on autoshaping in the pigeon). Thus, both previous results and those of the present study lead to the conclusion that probability of the US per se does not induce the pattern of results noted in this study. The finding that sign-tracking was reduced, and was accompanied by increases in goaltracking in some subjects in the second experiment, is explicable only in terms of the negative response contingency itself.

\section{REFERENCES}

Azrin, N. H., \& HaKE, D. F. Positive conditioned suppression: Conditioned suppression using positive reinforcers as the unconditioned stimuli. Journal of the Experimental Analysis of Behavior, 1969, 12, 167-173.

Barrera, F. J. Centrifugal selection of signal directed pecking. Journal of the Experimental Analysis of Behavior, 1974, 22, 341-355. 
Boakes, R. A. Performance on learning to associate a stimulus with positive reinforcement. In H. Davis \& H. M. B. Hurwitz (Eds.), Operant-Pavlovian interactions. Hillsdale, N.J: Erlbaum, 1977.

BoAKes, R. A. Interactions between Type I and Type II processes involving positive reinforcement. In $\mathbf{A}$. Dickinson \& $\mathrm{R}$. $\mathbf{A}$. Boakes (Eds.), Mechanisms of learning and motivation: $A$ memorial volume to Jerzy Konorski. Hillsdale, N.J: Erlbaum, 1979.

Brown, P. L., \& Jenkins, H. Autoshaping of the pigeon's keypeck. Journal of the Experimental Analysis of Behavior, 1968, 11, 1-8.

Dickinson, A., \& Dearing, M. F. Appetitive-aversive interactions and inhibitory processes. In A. Dickinson \& R. A. Boakes (Eds.), Mechanisms of learning and motivation: $A$ memorial volume to Jerzy Konorski. Hillsdale, N.J: Erlbaum, 1979.

Dickinson, A., \& Mackintosh, N. J. Classical conditioning in animals. In M. R. Rosenzweig \& L. W. Porter (Eds.), Annual review of psychology. Palo Alto, Calif: Annual Reviews, 1978.

Dunham, P. J. Punishment: Method and theory. Psychological Review, 1971, 78, 58-80.

Fleshler, M., \& Hoffman, H. S. A progression for generating variable interval schedules. Journal of the Experimental Analysis of Behavior, 1962, 5, 529-530.

GamzU, E., \& Schwam, E. Autoshaping and automaintenance of a key-press response in squirrel monkeys. Journal of the Experimental Analysis of Behavior, 1974, 21, 361-371.

Gibbon, J., Farkell, L., Locurto, C. M., Duncan, H. J., \& Terrace, H. S. Partial reinforcement in autoshaping with pigeons. Animal Learning \& Behavior, 1980, 8, $45-59$.

Gutman, A., \& Maie r, S. F. Operant and Pavlovian factors in cross-response transfer of inhibitory stimulus control. Learning and Motivation, 1978, 9, 231-254.

Hearst, E., \& Jenkins, H. M. Sign tracking: The stimulusreinforcer relation and directed action. Austin, Tex: Psychonomic Society, 1974.

Holland, P. C. Conditioned stimulus as a determinant of the form of the Pavlovian conditioned response. Journal of Experimental Psychology: Animal Behavior Processes, 1977, 3, 77-104.

Holland, P. C. Differential effects of omission contingencies on various components of Pavlovian appetitive conditioned responding in rats. Journal of Experimental Psychology: Animal Behavior Processes, 1979, 5, 178-193.

Jenkins, H. M. Sensitivity of different response systems to stimulus-reinforcer and response-reinforcer relations. In H. Davis \& H. M. B. Hurwitz (Eds.), Operant-Pavlovian interactions. Hillsdale, N.J: Erlbaum, 1977.

KARPICKE, J. Directed approach responses and positive conditioned suppression in the rat. Animal Learning \& Behavior, $1978,6,216-224$.

Karpicke, J., Christoph, C., Peterson, G., \& Hearst, E. Signal location and positive vs, negative conditioned suppression in the rat. Journal of Experimental Psychology: Animal Behavior Processes, 1977, 3, 105-118.
LAJOIE, J., \& Bindra, D. An interpretation of autoshaping and related phenomena in terms of stimulus-incentive contingencies alone. Canadian Journal of Psychology, 1976, 30, 157-173.

Locurto, C. M. Contributions of autoshaping to the partitioning of conditioned behavior. In C. M. Locurto, H. S. Terrace, \& J. Gibbon (Eds.), Autoshaping and conditioning theory. New York: Academic Press, 1980.

Locurto, C. M., Duncan, H., Terrace, H. S., \& Gibbon, J. Autoshaping in the rat: Interposing delays between responses and food. Animal Learning \& Behavior, 1980, 8, 37-44.

Locurto, C., Terrace, H. S., \& GibBon, J. Autoshaping, random control, and omission training in the rat. Journal of the Experimental Analysis of Behavior, 1976, 25, 451-462.

Locurto, C. M., Terrace, H. S., \& Gibbon, J. Omission training (negative automaintenance) in the rat: Effects of trial offset. Bulletin of the Psychonomic Society, 1978, 12, 11-14.

LuCAS, G. A. The control of keypecks during automaintenance by prekeypeck omission training. Animal Learning \& Behavior, 1975, 3, 33-36.

Peden, B. F., Browne, M. P., \& Hearst, E. Persistent approaches to a signal for food despite food omission for approaching. Journal of Experimental Psychology: Animal Behavior Processes, 1977, 3, 377-399.

Poling, A., \& Poling, T. Automaintenance in guinea pigs: Effects of feeding regimen and omission training. Journal of the Experimental Analysis of Behavior, 1978, 30, 37-46.

Powell, R. W., Kelly, W., \& Santisteban, D. Responseindependent reinforcement in the crow: Failure to obtain autoshaping or positive automaintenance. Bulletin of the Psychonomic Society, 1975, 6, 513-516.

Schwartz, B. Positive and negative conditioned suppression in the pigeon: Effects of the locus and modality of the CS. Learning and Motivation, 1976, 7, 86-100.

Schwartz, B., \& Gamzu, E. Pavlovian control of operant behavior: An analysis of autoshaping and its implications for operant conditioning. In W. K. Honig \& J. E. R. Staddon (Eds.), Handbook of operant behavior. Englewood Cliffs, N.J: Prentice-Hall, 1977.

Staddon, J. E. R. Behavioral competition in conditioning situations: Notes toward a theory of generalization and inhibition. In H. Davis \& H. M. B. Hurwitz (Eds.), Operant-Pavlovian interactions. Hillsdale, N.J: Erlbaum, 1977. (a)

Staddon, J. E. R. Schedule-induced behavior. In W. K. Honig \& J, E. R. Staddon (Eds.), Handbook of operant behavior. New York: Appleton-Century-Crofts, 1977. (b)

Williams, D. R., \& Williams, H. Auto-maintenance in the pigeon: Sustained pecking despite contingent nonreinforcement. Journal of the Experimental Analysis of Behavior, 1969, 12, 511-520.

Woodruff, G., \& Williams, D. R. The associative relation underlying autoshaping in the pigeon. Journal of the Experimental Analysis of Behavior, 1976, 26, 1-13.

(Received for publication August 8, 1980; revision accepted January 20,1981 .) 